\title{
Portfolio Strategy of Financial Market with Regime Switching Driven by Geometric Lévy Process
}

\author{
Liuwei Zhou and Zhijie Wang \\ College of Information Science and Technology, Donghua University, Shanghai 201620, China \\ Correspondence should be addressed to Liuwei Zhou; zhouliuwei@hotmail.com
}

Received 18 January 2014; Accepted 24 February 2014; Published 25 March 2014

Academic Editor: Zhengguang Wu

Copyright ( 2014 L. Zhou and Z. Wang. This is an open access article distributed under the Creative Commons Attribution License, which permits unrestricted use, distribution, and reproduction in any medium, provided the original work is properly cited.

The problem of a portfolio strategy for financial market with regime switching driven by geometric Lévy process is investigated in this paper. The considered financial market includes one bond and multiple stocks which has few researches up to now. A new and general Black-Scholes (B-S) model is set up, in which the interest rate of the bond, the rate of return, and the volatility of the stocks vary as the market states switching and the stock prices are driven by geometric Lévy process. For the general B-S model of the financial market, a portfolio strategy which is determined by a partial differential equation (PDE) of parabolic type is given by using Itô formula. The PDE is an extension of existing result. The solvability of the PDE is researched by making use of variables transformation. An application of the solvability of the PDE on the European options with the final data is given finally.

\section{Introduction}

To make a portfolio strategy is to search for a best allocation of wealth among different assets in markets. Taking the European options, for instance, how to distribute the appropriate proportions of each option to maximize total returns at expire time is the core of portfolio strategy problem. There are two points mentioned among the relevant literatures for portfolio selection problems: setting up a market model that approximates to the real financial market and the way of solving it.

Portfolio strategy researches are based on portfolio selection analysis given by Markowitz [1]. Extension of Markowitz's work to the multiperiod model has given by $\mathrm{Li}$ and $\mathrm{Ng}$ [2] which derived the analytical optimal portfolio policy. These previous researches were assuming that the underlying market has only one state or mode. But the real market might have more than one state and could switch among them. Then, portfolio policies under regime switching have been widely discussed. In a financial market model, the key process $S$ that models the evolution of stock price should be a Brownian motion. Indeed, this can be intuitively justified on the basis of the central limit theorem if one perceives the movement of stocks. The analysis of Øksendal [3] was mainly based on the generalized Black-Scholes model which has two assets $B(t)$ and $S(t)$ as $d B(t)=\rho(t) B(t) d t$ and $d S(t)=\alpha(t) S(t) d t+\beta(t) S(t) d W(t)$, where $W(t)$ is a Brownian motion. In that case, Øksendal formulated optimal selling decision making as an optimal stopping problem and derived a closed-form solution. The underlying problem may be treated as a free boundary value problem, which was extended to incorporate possible regime switching by Guo and Zhang [4] and Pemy et al. [5] with the switching represented by a two-state Markov chain. The rate of return $\alpha(\mathrm{t})$ in the above Black-Scholes models in $[4,5]$ is a Markov chain which is different from the general one. As an application, $\mathrm{Wu}$ and $\mathrm{Li}$ $[6,7]$ have given the strategy of multiperiod mean-variance portfolio selection with regime switching and a stochastic cash flow which depends on the states of a stochastic market following a discrete-time Markov chain. Being put in the Markov jump, Black-Scholes model with regime switching is much closer to the real market.

In recent years, Lévy process as a more general process than Brownian motion has been applied in financial portfolio 
optimization. Kallsen [8] gave an optimal portfolio strategy of securities market under exponential Lévy process. More specific than exponential Lévy process, a financial market model with stock price following the geometric Lévy process was discussed by Applebaum [9] in which a Lévy process $X(t)$ and geometric Lévy motion $S(t)=e^{X(t)}$ were introduced. Taking $X$ to be a Lévy process could force our stock prices clearly not moving continuously, and a more realistic approach is that the stock price is allowed to have small jumps in small time intervals. Some applications of financial market driven by Lévy process are taken on life insurance. Vandaele and Vanmaele [10] show the real riskminimizing hedging strategy for unit-linked life insurance in financial market driven by a Lévy process while Weng [11] has analyzed the constant proportion portfolio insurance by assuming that the risky asset price follows a regime switching exponential Lévy process and obtained the analytical forms of the shortfall probability, expected shortfall and expected gain. Optimizing proportional reinsurance and investment policies in a multidimensional Lévy-driven insurance model is discussed by Bäuerle and Blatter [12]. Moreover, under a generally method, Yuen and Yin [13] have considered the optimal dividend problem for the insurance risk process in a general Lévy process which shows that if the Lévy density is a completely monotone function, then the optimal dividend strategy is a barrier strategy.

Among all the above literatures, those portfolios are always based on one risk-free asset and only one risky asset which may limit the chosen stocks. However, in a real financial market, there always exists more than one risky asset in a portfolio. That is why we are going to extend the singlestock financial market model to a multistock financial market model driven by geometric Lévy process which is more closer to the real market than proposed portfolios cited above. In this paper, we set up a general Black-Scholes model with geometric Lévy process. For the general Black-Scholes model of the financial market, a portfolio strategy which is determined by a partial differential equation (PDE) of parabolic type is given by using Itô formula. The solvability of the PDE is researched by making use of variables transformation. An application of the solvability of the PDE on the European options with the final data is given finally. The contributions of this paper are as follows. (i) The B-S market model is extended into general form in which the interest rate of the bond, the rate of return, and the volatility of the stock vary as the market states switching and the stock prices are driven by geometric Lévy process. (ii) The PDE determining the portfolio strategy and its solvability are extensions of the existing results.

\section{Problem Formulation}

Assume that $(\Omega, \mathscr{F}, P)$ is a complete probability space and $\left\{\mathscr{F}_{t}: t \geq 0\right\}$ is a nondecreasing family of $\sigma$-algebra subfields of $\mathscr{F} .\{\alpha(t): t \geq 0\}$ denotes a Markov chain in $(\Omega, \mathscr{F}, P)$ as the regime of financial market, for example, the bull market or bear market of a stock market. Let $M=\{1,2, \ldots, m\}$ be the regime space of this Markov chain, and let $\Gamma=\left(\gamma_{i j}\right)_{m \times m}$ be the transition rate matrix which is satisfying

$$
P\{\alpha(t+\Delta)=j \mid \alpha(t)=i\}= \begin{cases}\gamma_{i j} \Delta+o(\Delta), & i \neq j, \\ 1+\gamma_{i i} \Delta+o(\Delta), & i=j,\end{cases}
$$

where $\Delta>0$ is the increment of time, $\gamma_{i j} \geq 0(i \neq j), \gamma_{i i}=$ $-\sum_{j \neq i, j=1}^{m} \gamma_{i j}$.

In this paper, we consider a financial market model driven by geometric Lévy process. The market consists of one risk-free asset denoted by $B$ and $n$ risky assets denoted by $S_{1}, S_{2}, \ldots, S_{n}$. The price process of these assets obeys the following dynamic equations in which the price process of the risky assets follows the geometric Lévy process; that is,

$$
\begin{gathered}
d B(t)=B(t) r(t, \alpha(t)) d t, \quad B(0)=B_{0}, \\
d S_{k}(t)=S_{k}(t)\left[\mu_{k}(t, \alpha(t)) d t+\sigma_{k}(t, \alpha(t)) d W_{k}(t)\right. \\
\left.+\int_{R-\{0\}} z \widetilde{N}_{k}(d t, d z)\right], \\
S_{k}(0)=S_{k}^{0}>0,
\end{gathered}
$$

where $B(t)$ is the price of $B$ with the interest rate $r(t, \alpha(t))$ and $S_{k}(t)$ is the price of $S_{k}$ with the expect rate of return $\mu_{k}(t, \alpha(t))$ and the volatility $\sigma_{k}(t, \alpha(t))$, which follow the regime switching of financial market. $S_{1}(t), S_{2}(t), \ldots, S_{n}(t)$ are independent from each other. $W_{k}(t)$ is the Brownian motion which is independent from $\{\alpha(t): t \geq 0\} . \widetilde{N}_{k}(\cdot, \cdot)$ is defined as below

$$
\widetilde{N}_{k}(d t, d z)=N_{k}(d t, d z)-\eta_{k}(d z) d t,
$$

where $N_{k}(d t, d z)$ and $\eta_{k}(d z) d t$ indicate the number of jumps and average number of jumps within time $d t$ and jump range $d z$ of price process $S_{k}(t)$, respectively. That is

$$
\eta_{k}(d z) d \mathrm{t}=\mathbb{E}\left[N_{k}(d t, d z)\right],
$$

where $\mathbb{E}$ is the expectation operator. Moreover, we assume that $N_{k}(d t, d z), \alpha(t)$, and $W_{k}(t)(k=1,2, \ldots, n)$ are independent of each other.

Remark 1. The finance market model (2) is an extension of the B-S market model in which the interest rate of the bond, the rate of return, and the volatility of the stock vary as the market states switching and the stock prices are driven by geometric Lévy process.

For finance market model (2), we introduce the concept of self-financing portfolio as follows.

Definition 2. A self-financing portfolio $(\varphi, \psi)=\left(\varphi, \psi_{1}\right.$, $\left.\psi_{2}, \ldots, \psi_{n}\right)$ for the financial market model (2) is a series of predictable processes

$$
\{\varphi(t)\}_{t \geq 0}, \quad\left\{\psi_{k}(t)\right\}_{t \geq 0} \quad(k=1,2, \ldots, n),
$$


that is, for each $T>0$,

$$
\int_{0}^{T}|\varphi(s)|^{2} d s+\sum_{k=1}^{n} \int_{0}^{T}\left|\psi_{k}(s)\right|^{2} d s<\infty
$$

and the corresponding wealth process $\{V(t)\}_{t \geq 0}$, defined by

$$
V(t):=\varphi(t) B(t)+\sum_{k=1}^{n} \psi_{k}(t) S_{k}(t), \quad t \geq 0,
$$

is an Itô process satisfying

$$
d V(t)=\varphi(t) d B(t)+\sum_{k=1}^{n} \psi_{k}(t) d S_{k}(t), \quad t \geq 0 .
$$

Problem Formulation. In this note, we will propose a portfolio strategy for the financial market model (2) which is determined by a partial differential equation (PDE) of parabolic type by using Itô formula. The solvability of the PDE is researched by making use of variables transformation. Furthermore, the relationship between the solution of the $\mathrm{PDE}$ and the wealth process will be discussed.

\section{Main Results and Proofs}

In this section, we will give the following fundamental results. For the sake of simplification, we write $r(t, \alpha(t))$ as $r, f(t, S(t))$ as $f$, and so forth.

To obtain the main result, we give the solution of (2) and the characteristic of the derivation (8) of the wealth process.

The exact solutions of $B(t)$ in (2) can be found as follows:

$$
B(t)=B(0) \exp \left(\int_{0}^{t} r(s, \alpha(s)) d s\right) .
$$

To solve the second equation in (2) for $S_{k}(t)$, it follows from the Itô formula that

$$
\begin{aligned}
d \ln S_{k}(t)= & \frac{1}{S_{k}(t)}\left[S_{k}(t) \mu_{k}(t, \alpha(t)) d t\right. \\
& \left.+S_{k}(t) \sigma_{k}(t, \alpha(t)) d W_{k}(t)\right] \\
& -\frac{1}{2} \frac{1}{S_{k}^{2}(t)} S_{k}^{2}(t) \sigma_{k}^{2}(t, \alpha(t)) d t \\
& +\int_{R-\{0\}}\left[\ln \left(S_{k}(t)+z S_{k}(t)\right)\right. \\
& +\int_{R-\{0\}}\left[\ln \left(S_{k}(t)+z S_{k}(t)\right)-\ln \left(S_{k}(t)\right)\right. \\
& +\sum_{j=1}^{s} \gamma_{i j} \ln \left(S_{k}(t)\right) \\
& \left.-z S_{k}(t) \frac{1}{S_{k}(t)}\right] \eta_{k}(d z) d t
\end{aligned}
$$

$$
\begin{aligned}
= & {\left[\mu_{k}(t, \alpha(t))-\frac{1}{2} \sigma_{k}^{2}(t, \alpha(t))\right] d t } \\
& +\sigma_{k}(t, \alpha(t)) d W_{k}(t) \\
& +\int_{R-\{0\}} \ln (1+z) \widetilde{N}_{k}(d t, d z) \\
& +\int_{R-\{0\}}[\ln (1+z)-z] \eta_{k}(d z) d t .
\end{aligned}
$$

Integrating both sides of the above equation from 0 to $t$, we have

$$
\begin{aligned}
S_{k}(t)=S_{k}^{0} \exp \left\{\int_{0}^{t}\left(\mu_{k}(s, \alpha(s))-\frac{1}{2} \sigma_{k}^{2}(s, \alpha(s))\right] d s\right. \\
+\int_{0}^{t} \sigma_{k}(s, \alpha(s)) d W_{k}(s) \\
+\int_{0}^{t} \int_{R-\{0\}} \ln (1+z) \widetilde{N}_{k}(d s, d z) \\
\left.+\int_{0}^{t} \int_{R-\{0\}}[\ln (1+z)-z] \eta_{k}(d z) d s\right\} .
\end{aligned}
$$

Proposition 3. Consider the price model (2) of a financial market. If a portfolio $(\varphi, \psi)$ is a self-financing strategy, then the wealth process $\{V(t)\}_{t \geq 0}$ defined by (7) satisfies

$$
\begin{aligned}
d V(t)= & \{r(t, \alpha(t)) V(t) \\
& +\sum_{k=1}^{n} \psi_{k}(t) S_{k}(t)\left[\mu_{k}(t, \alpha(t))-r(t, \alpha(t))\right. \\
& \left.\left.\quad-\int_{R-\{0\}} z \eta_{k}(d z)\right]\right\} d t \\
& +\sum_{k=1}^{n} \psi_{k}(t) S_{k}(t) \sigma_{k}(t, \alpha(t)) d W_{k}(t) \\
& +\sum_{k=1}^{n} \psi_{k}(t) S_{k}(t) \int_{R-\{0\}} z N_{k}(d t, d z) .
\end{aligned}
$$

Conversely, consider the model (2) of a financial market. If a pair $(\varphi, \psi)$ of predictable processes following the wealth process $\{V(t)\}_{t \geq 0}$ defined by formula (7) satisfies $(12)$, then $(\varphi, \psi)$ is a self-financing strategy.

Proof. Substituting (2) into (8), we have

$$
\begin{aligned}
d V(t) & =\varphi(t) d B(t)+\sum_{k=1}^{n} \psi_{k}(t) d S_{k}(t) \\
& =\varphi(t) B(t) r(t, \alpha(t)) d t+\sum_{k=1}^{n} \psi_{k}(t) S_{k}(t)
\end{aligned}
$$




$$
\begin{aligned}
& \times\left[\mu_{k}(t, \alpha(t)) d t+\sigma_{k}(t, \alpha(t)) d W_{k}(t)\right. \\
& \left.+\int_{R-\{0\}} z \widetilde{N}_{k}(d t, d z)\right] \\
& =\left\{\left[V(t)-\sum_{k=1}^{n} \psi_{k}(t) S_{k}(t)\right] r(t, \alpha(t))\right. \\
& \left.+\sum_{k=1}^{n} \psi_{k}(t) S_{k}(t) \mu_{k}(t, \alpha(t))\right\} d t \\
& +\sum_{k=1}^{n} \psi_{k}(t) S_{k}(t) \sigma_{k}(t, \alpha(t)) d W_{k}(t) \\
& +\sum_{k=1}^{n} \psi_{k}(t) S_{k}(t) \int_{R-\{0\}} z \widetilde{N}_{k}(d t, d z) \\
& =\left\{r(t, \alpha(t)) V(t)+\sum_{k=1}^{n} \psi_{k}(t) S_{k}(t)\right. \\
& \times\left[\mu_{k}(t, \alpha(t))-r(t, \alpha(t))\right. \\
& \left.\left.-\int_{R-\{0\}} z \eta_{k}(d z)\right]\right\} d t \\
& +\sum_{k=1}^{n} \psi_{k}(t) S_{k}(t) \sigma_{k}(t, \alpha(t)) d W_{k}(t) \\
& +\sum_{k=1}^{n} \psi_{k}(t) S_{k}(t) \int_{R-\{0\}} z N_{k}(d t, d z),
\end{aligned}
$$

which is (12).

Conversely, from (2) and (12), we can obtain (8).

This completes the proof of the above proposition.

Now we give the following fundamental results.

Theorem 4. Consider the model (2) of a financial market. Assume that the portfolio $\left(\varphi, \psi_{1}, \psi_{2}, \ldots, \psi_{n}\right)$ is a self-financing strategy and $\{V(t)\}_{t \geq 0}$ is the wealth process defined by (7) and $\sum_{k=1}^{n} \psi_{k} S_{k} \int_{R-\{0\}} z \eta_{k}(d z)=\sum_{k=1}^{n} \int_{R-\{0\}} z \psi_{k} S_{k} \eta_{k}(d z)$. If there exists a function $f(t, S)$ of $C^{1,2}$ class (the set of functions which are once differentiable in t and continuously twice differentiable in S) such that

$$
\begin{aligned}
& V(t)=f(t, S(t)), \quad t \in[0, T], \\
& S(t)=\left(S_{1}(t), S_{2}(t), \ldots, S_{n}(t)\right),
\end{aligned}
$$

which holds true, then the portfolio $\left(\varphi, \psi_{1}, \psi_{2}, \ldots, \psi_{n}\right)$ satisfies

$$
\begin{gathered}
\varphi(t)=\frac{f-(\partial f / \partial S) S^{T}}{B(t)}, \quad t \geq 0 \\
\psi(t)=\left(\frac{\partial f}{\partial S_{1}}, \frac{\partial f}{\partial S_{2}}, \ldots, \frac{\partial f}{\partial S_{n}}\right)=\frac{\partial f}{\partial S}, \quad t \geq 0
\end{gathered}
$$

and the function $f(t, S)$ solves the following backward PDE of parabolic type:

$$
\begin{array}{r}
\frac{\partial f}{\partial t}+r \sum_{k=1}^{n} \frac{\partial f}{\partial S_{k}} S_{k}+\frac{1}{2} \sum_{i=1}^{n} \sum_{j=1}^{n} \frac{\partial^{2} f}{\partial S_{i} \partial S_{j}} S_{i} \sigma_{i} S_{j} \sigma_{j}=r f, \\
t<T, S>0 .
\end{array}
$$

Moreover, if $V(T)=g(S(T))$, then the function $f(t, S)$ satisfies the following equation:

$$
f(T, S)=g(S), \quad S>0 .
$$

For the converse part, we assume that $T>0$. If there exists a function $f(t, S)$ of $C^{1,2}$ class such that (17) and (18) are satisfied, then the process $(\varphi, \psi)$ defined by (16) and (15) is a self-financing strategy. The wealth process $V=\{V(t)\}_{t \in[0, T]}$ corresponding to $(\varphi, \psi)$ satisfies (14).

Proof. We proof the direct part of Theorem 4 firstly.

For

$$
V(t)=f(t, S(t)),
$$

by applying the Itô formula, we can infer that

$$
\begin{aligned}
d V(t)= & \frac{\partial f}{\partial t}(t, S(t)) d t \\
& +\sum_{k=1}^{n} \frac{\partial f}{\partial S_{k}}(t, S(t))\left(S_{k} \mu_{k} d t+S_{k} \sigma_{k} d W_{k}\right) \\
& +\frac{1}{2} \sum_{i=1}^{n} \sum_{j=1}^{n} \frac{\partial^{2} f}{\partial S_{i} \partial S_{j}}(t, S(t)) S_{i} \sigma_{i} S_{j} \sigma_{j} d t \\
& +\sum_{k=1}^{n} \int_{R-\{0\}}(f(t, S+z S)-f(t, S)) \widetilde{N}_{k}(d t, d z) \\
& +\sum_{k=1}^{n} \int_{R-\{0\}}[f(t, S+z S)-f(t, S) \\
& +\sum_{k=1}^{n} \frac{\partial f}{\partial S_{k}} S_{k} \sigma_{k} d W_{k} \\
& +\sum_{j=1}^{n} \gamma_{i j} f(t, S(t)) \\
& \left.-\sum_{k=1}^{n} \int_{R-\{0\}} z \frac{\partial f}{\partial S_{k}} S_{k} \eta_{k}(d z)\right] d t \\
& {\left[\begin{array}{l}
\partial t \\
\end{array} \sum_{k=1}^{n} \frac{\partial f}{\partial S_{k}} S_{k} \mu_{k}+\frac{1}{2} \sum_{i=1}^{n} \sum_{j=1}^{n} \frac{\partial^{2} f}{\partial S_{i} \partial S_{j}} S_{i} \sigma_{i} S_{j} \sigma_{j}\right.} \\
& \\
& \left.+t, S) S_{k}\right] \eta_{k}(d z) d t
\end{aligned}
$$


On the other hand, since our strategy is self-financing, the formula (12) is satisfied.

Thus, the rate of return and the volatility in (20) and (12) should be coincided, and hence

$$
\begin{gathered}
\sum_{k=1}^{n} \psi_{k}(t) S_{k}(t) \sigma_{k}=\sum_{k=1}^{n} \frac{\partial f}{\partial S_{k}}(t, S) S_{k} \sigma_{k}, \\
r(t, \alpha(t)) f(t, S)+\sum_{k=1}^{n} \psi_{k} S_{k}\left(\mu_{k}-r\right) \\
=\frac{\partial f}{\partial t}+\sum_{k=1}^{n} \frac{\partial f}{\partial S_{k}} S_{k} \mu_{k}+\frac{1}{2} \sum_{i=1}^{n} \sum_{j=1}^{n} \frac{\partial^{2} f}{\partial S_{i} \partial S_{j}} S_{i} \sigma_{i} S_{j} \sigma_{j} .
\end{gathered}
$$

We can easily get $S_{k} \geq 0$ from (11), which together with the first equation of (21) and the independence of $S_{k} \quad(k=$ $1,2, \ldots, n)$ yields $(16)$.

From the first equation of (21), (7), and (14), we have

$$
r \varphi B=f-\sum_{k=1}^{n} \frac{\partial f}{\partial S_{k}} S_{k} .
$$

So that

$$
\varphi=\frac{f-\sum_{k=1}^{n}\left(\partial f / \partial S_{k}\right) S_{k}}{B}=\frac{f-f_{S} S^{T}}{B} .
$$

Substituting (16) into the second equation of (21), we have

$$
r f-\sum_{k=1}^{n} \psi_{k} S_{k} r=\frac{\partial f}{\partial t}+\frac{1}{2} \sum_{i=1}^{n} \sum_{j=1}^{n} \frac{\partial^{2} f}{\partial S_{i} \partial S_{j}} S_{i} \sigma_{i} S_{j} \sigma_{j},
$$

which is (17).

Conversely, assume that $f=f(t, S)$ is a $C^{1,2}$-class function which is a solution of the $\operatorname{PDE}(17)$, and that $(\varphi, \psi)$ is a process defined by (16) and (15).

Firstly, we will show that a process $V=V(t), t \in[0, T]$, defined by (7) satisfies the equation:

$$
V(t)=f(t, S(t)), \quad t \in[0, T] .
$$

In fact, substituting formulas (16) and (15) into the right hand side of (7), we have

$$
\begin{aligned}
V(t)= & \varphi B+\sum_{k=1}^{n} \psi_{k} S_{k}=\frac{f-\sum_{k=1}^{n}\left(\partial f / \partial S_{k}\right) S_{k}}{B} B \\
& +\sum_{k=1}^{n} \frac{\partial f}{\partial S_{k}} S_{k}=f, \quad t \geq 0 .
\end{aligned}
$$

This proves (25).

Next, we will show that $(\varphi, \psi)$ is a self-financing strategy; that is, (12) holds.

By applying the Itô formula to the process $V$ and function $f$, we have that $(20)$ is satisfied.
Furthermore, by (17),

$$
\begin{gathered}
\frac{\partial f}{\partial t}+\frac{1}{2} \sum_{i=1}^{n} \sum_{j=1}^{n} \frac{\partial^{2} f}{\partial S_{i} \partial S_{j}} S_{i} \sigma_{i} S_{j} \sigma_{j}=r f-r \sum_{k=1}^{n} S_{k} \frac{\partial f}{\partial S_{k}}, \\
\frac{\partial f}{\partial t}+\sum_{k=1}^{n} S_{k} \mu_{k} \frac{\partial f}{\partial S_{k}}+\frac{1}{2} \sum_{i=1}^{n} \sum_{j=1}^{n} \frac{\partial^{2} f}{\partial S_{i} \partial S_{j}} S_{i} \sigma_{i} S_{j} \sigma_{j} \\
=r f+\sum_{k=1}^{n}\left(\mu_{k}-r\right) S_{k} \frac{\partial f}{\partial S_{k}} .
\end{gathered}
$$

Then, by (25) and (16), we have

$$
\begin{aligned}
r V+\sum_{k=1}^{n} \psi_{k} S_{k}\left(\mu_{k}-r\right)= & \frac{\partial f}{\partial t}+\sum_{k=1}^{n} S_{k} \mu_{k} \frac{\partial f}{\partial S_{k}} \\
& +\frac{1}{2} \sum_{i=1}^{n} \sum_{j=1}^{n} \frac{\partial^{2} f}{\partial S_{i} \partial S_{j}} S_{i} \sigma_{i} S_{j} \sigma_{j}, \\
\sum_{k=1}^{n} \psi_{k} S_{k} \sigma_{k} & =\sum_{k=1}^{n} \frac{\partial f}{\partial S_{k}} S_{k} \sigma_{k} .
\end{aligned}
$$

Those together with (16) yield that (20) implies (12). The proof of Theorem 4 is completed.

Remark 5. In order to determine the portfolio strategy $(\phi, \psi)$ and obtain the final value $V(t)$, from Theorem 4 , we should find the solution of the PDF (17) with the final data (18). This is the key problem in the rest of this section. We have the following result in terms of method of variables transformation.

Theorem 6. Let $r(t, \alpha(t))$ in (2) be a constant $r$. The function $f(t, S), t \leq T, S>0$ given by the following formula:

$$
\begin{aligned}
& f(t, S)= \frac{e^{-r(T-t)}}{\sqrt{2 \pi}} \\
& \times \sum_{i=1}^{n} \int_{-\infty}^{\infty} e^{-x_{i}^{2} / 2} g\left(0, \ldots, 0, S_{i} e^{\sigma_{i} \sqrt{T-t} x_{i}-\left(r-\sigma_{i}^{2} / 2\right)(t-T)},\right. \\
&0, \ldots, 0) d x_{i},
\end{aligned}
$$

is a solution of the general Black-Scholes equation (17) with the final data (18).

Proof. We are going to do some equivalent transformations of general B-S equation (17), in order to get an appropriate equivalent equation with analytic solutions. The procedure will be divided into four steps.

Step I. Let

$$
\begin{gathered}
f\left(t, S_{1}, \ldots, S_{n}\right)=e^{r(t-T)} q\left(t, \ln S_{1}-\left(r-\frac{1}{2} \sigma_{1}^{2}\right)(t-T), \ldots,\right. \\
\left.\ln S_{n}-\left(r-\frac{1}{2} \sigma_{n}^{2}\right)(t-T)\right),
\end{gathered}
$$


and denote $y_{i}=\ln S_{i}-\left(r-(1 / 2) \sigma_{i}^{2}\right)(t-T)(i=1,2, \ldots, n)$, and then

$$
\begin{aligned}
& \frac{\partial f}{\partial t}= \frac{d\left(e^{r(t-T)}\right)}{d t} q+e^{r(t-T)} q_{t} \\
&= e^{r(t-T)} q\left(\frac{d r}{d t}(t-T)+r\right) \\
&+e^{r(t-T)}\left[q_{t}-\sum_{i=1}^{n} \frac{\partial q}{\partial y_{i}}\left(r-\frac{1}{2} \sigma_{i}^{2}\right)\right] \\
&= r e^{r(t-T)} q+e^{r(t-T)} q \frac{d r}{d t}(t-T) \\
&+e^{r(t-T)}\left[\frac{\partial q}{\partial t}-\sum_{i=1}^{n} \frac{\partial q}{\partial y_{i}}\left(r-\frac{1}{2} \sigma_{i}^{2}\right)\right], \\
& \frac{\partial^{2} f}{\partial S_{i} \partial S_{j}}= \frac{\partial f}{\partial S_{i}}=e^{r(t-T)} \frac{\partial q}{\partial y_{i}} \frac{1}{S_{i}}, \\
&=\left\{\begin{array}{l}
\left.e^{r(t-T)}\left(\partial q / \partial y_{i}\right)\left(1 / S_{i}\right)\right) \\
\partial S_{j}
\end{array}\right. \\
& e^{r(t-T)}\left(\frac{\partial^{2} q}{\partial y_{i} \partial y_{j}} \frac{1}{\partial y_{i} \partial y_{i}} \frac{1}{S_{j}}, \frac{1}{S_{i}} \frac{1}{S_{i}}-\frac{\partial q}{\partial y_{i}} \frac{1}{S_{i}^{2}}\right), \quad i=j .
\end{aligned}
$$

Inserting the above formulas into (17), we get

$$
\begin{aligned}
r f & +\frac{d r}{d t}(t-T) q e^{r(t-T)}+e^{r(t-T)}\left[\frac{\partial q}{\partial t}-\sum_{i=1}^{n} \frac{\partial q}{\partial y_{i}}\left(r-\frac{1}{2} \sigma_{i}^{2}\right)\right] \\
& +r \sum_{i=1}^{n} e^{r(t-T)} \frac{\partial q}{\partial y_{i}} \frac{1}{S_{i}} S_{i}+\frac{1}{2} \sum_{i=1}^{n} \sum_{j=1}^{n} e^{r(t-T)} \frac{\partial^{2} q}{\partial y_{i} \partial y_{j}} \frac{1}{S_{i}} \frac{1}{S_{j}} S_{i} \sigma_{i} S_{j} \sigma_{j} \\
& -\frac{1}{2} \sum_{i=1}^{n} e^{r(t-T)} \frac{\partial q}{\partial y_{i}} S_{i}^{2} \frac{1}{S_{i}^{2}} S_{i}^{2}=r f
\end{aligned}
$$

which can be simplified as

$$
\frac{d r}{d t}(t-T) q+\frac{\partial q}{\partial t}+\frac{1}{2} \sum_{i=1}^{n} \sum_{j=1}^{n} \frac{\partial^{2} q}{\partial y_{i} \partial y_{j}} \sigma_{i} \sigma_{j}=0 .
$$

The final data $f(T, S)=g(S)$ can be rewritten as

$$
q(T, S)=g\left(e^{S_{1}}, e^{S_{2}}, \ldots, e^{S_{n}}\right) .
$$

Step II. We introduce another variable and a new function as follows:

$$
\begin{gathered}
\tau=T-t>0, \quad t=T-\tau, \tau \geq 0, t \leq T, \\
q(t, y)=u(T-t, y) \quad \text { or } \quad u(\tau, y)=q(T-\tau, y) .
\end{gathered}
$$

It can be computed that

$$
\begin{gathered}
q_{t}(t, y)=-u_{\tau}(T-t, y), \\
\frac{\partial q}{\partial y_{i}}=\frac{\partial u}{\partial y_{i}}(T-t, y), \\
\frac{\partial^{2} q}{\partial y_{i} \partial y_{j}}=\frac{\partial^{2} u}{\partial y_{i} \partial y_{j}}(T-t, y) .
\end{gathered}
$$

Substituting the above formulas into (34), we get

$$
\begin{gathered}
\frac{d V}{d t}(t-T) u(T-t, y)-u_{\tau}(T-t, y)+\frac{1}{2} \sum_{i=1}^{n} \sum_{j=1}^{n} \frac{\partial^{2} u}{\partial y_{i} \partial y_{j}} \sigma_{i} \sigma_{j} \\
=0, \\
u(0, y)=g\left(e^{y}\right) .
\end{gathered}
$$

Since $r(t, \alpha(t))$ is assumed as a constant $r$, (38) can be changed into

$$
\begin{gathered}
u_{\tau}(\tau, y)-\frac{1}{2} \sum_{i=1}^{n} \sum_{j=1}^{n} \frac{\partial^{2} u}{\partial y_{i} \partial y_{j}} \sigma_{i} \sigma_{j}=0, \\
u(0, y)=g\left(e^{y}\right) .
\end{gathered}
$$

Step III. We claim that the unique solution of (39) is

$$
\begin{aligned}
& u\left(t, y_{1}, y_{2}, \ldots, y_{n}\right) \\
& =\frac{1}{\sqrt{2 \pi \tau}} \sum_{i=1}^{n} \int_{-\infty}^{\infty} \frac{e^{-\left(y_{i}-x_{i}\right)^{2} / 2 \sigma_{i}^{2} \tau}}{\sigma_{i}} g\left(0, \ldots, 0, e^{x_{i}}, 0, \ldots, 0\right) d x_{i} .
\end{aligned}
$$

In fact,

$$
\begin{aligned}
u_{\tau}(\tau, y)= & -\frac{1}{2 \sqrt{2 \pi \tau} \tau} \\
& \times \sum_{i=1}^{n} \int_{-\infty}^{\infty} \frac{e^{-\left(y_{i}-x_{i}\right)^{2} / 2 \sigma_{i}^{2} \tau}}{\sigma_{i}} \\
& \times g\left(0, \ldots, 0, e^{x_{i}}, 0, \ldots, 0\right) d x_{i} \\
+\frac{1}{\sqrt{2 \pi \tau}} \sum_{i=1}^{n} \int_{-\infty}^{\infty} \frac{e^{-\left(y_{i}-x_{i}\right)^{2} / 2 \sigma_{i}^{2} \tau}}{\sigma_{i}} & \times g\left(0, \ldots, 0, e^{x_{i}}, 0, \ldots, 0\right) \\
& \times \frac{\left(y_{i}-x_{i}\right)^{2}}{2 \sigma_{i}^{2} \tau^{2}} d x_{i},
\end{aligned}
$$




$$
\begin{aligned}
& \frac{\partial u}{\partial y_{i}}=\frac{1}{\sqrt{2 \pi \tau}} \int_{-\infty}^{\infty} \frac{e^{-\left(y_{i}-x_{i}\right)^{2} / 2 \sigma_{i}^{2} \tau}}{\sigma_{i}} g\left(0, \ldots, 0, e^{x_{i}}, 0, \ldots, 0\right) \\
& \times\left(-\frac{y_{i}-x_{i}}{\sigma_{i}^{2} \tau}\right) d x_{i} \\
& \frac{\partial^{2} u}{\partial y_{i} \partial y_{j}}= \begin{cases}0, & i \neq j, \\
\frac{1}{\sqrt{2 \pi \tau}} \int_{-\infty}^{\infty} \frac{g\left(e^{x_{1}}, \ldots, e^{x_{n}}\right)}{\sigma_{i}^{2}} e^{-\left(y_{i}-x_{i}\right)^{2} / 2 \sigma_{i}^{2} \tau} & \\
\times\left(\frac{\left(y_{i}-x_{i}\right)^{2}}{\sigma_{i}^{4} \tau^{2}}-\frac{1}{\sigma_{i}^{2} \tau}\right) \sigma_{i}^{2} d x_{\mathrm{i}}, & i=j .\end{cases}
\end{aligned}
$$

So

$$
\begin{aligned}
u_{\tau}(\tau, g) & -\frac{1}{2} \sum_{i=1}^{n} \sum_{j=1}^{n} \frac{\partial^{2} u}{\partial y_{i} \partial y_{j}} \sigma_{i} \sigma_{j} \\
= & -\frac{1}{2 \sqrt{2 \pi \tau} \tau} \sum_{i=1}^{n} \int_{-\infty}^{\infty} \frac{e^{-\left(y_{i}-x_{i}\right)^{2} / 2 \sigma_{i}^{2} \tau}}{\sigma_{i}} \\
& \times g\left(0, \ldots, 0, e^{x_{i}}, 0, \ldots, 0\right) d x_{i} \\
& +\frac{1}{\sqrt{2 \pi \tau}} \sum_{i=1}^{n} \int_{-\infty}^{\infty} \frac{e^{-\left(y_{i}-x_{i}\right)^{2} / 2 \sigma_{i}^{2} \tau}}{\sigma_{i}} \\
& \times g\left(0, \ldots, 0, e^{x_{i}}, 0, \ldots, 0\right) \frac{\left(y_{i}-x_{i}\right)^{2}}{2 \sigma_{i}^{2} \tau^{2}} d x_{i} \\
& -\frac{1}{2} \sum_{i=1}^{n} \frac{1}{\sqrt{2 \pi \tau}} \int_{-\infty}^{\infty} \frac{g\left(e^{x_{1}}, \ldots, e^{x_{n}}\right)}{\sigma_{i}^{2}} \\
& \times e^{-\left(y_{i}-x_{i}\right)^{2} / 2 \sigma_{i}^{2} \tau}\left(\frac{\left(y_{i}-x_{i}\right)^{2}}{\sigma_{i}^{4} \tau^{2}}-\frac{1}{\sigma_{i}^{2} \tau}\right) \sigma_{i}^{2} d x_{i}=0 .
\end{aligned}
$$

Step IV. By introducing a change of variables $z_{i}=x_{i}-y_{i}$, we have $x_{i}=z_{i}+y_{i}$ and $d x_{i}=d z_{i}$, where $z_{i} \in(-\infty, \infty)$. It follows that

$$
\begin{aligned}
& u(\tau, y) \\
& \begin{aligned}
& \frac{1}{\sqrt{2 \pi \tau}} \sum_{i=1}^{n} \int_{-\infty}^{\infty} \frac{e^{-\left(y_{i}-x_{i}\right)^{2} / 2 \sigma_{i}^{2} \tau}}{\sigma_{i}} \\
& \times g\left(0, \ldots, 0, e^{z_{i}+y_{i}}, 0, \ldots, 0\right) d z_{i} .
\end{aligned}
\end{aligned}
$$

In order to get rid of the denominator $\sigma_{i}^{2} \tau$ in the exponent in the above formula, we make another change of variables as

$$
z_{i}=\sigma_{i} \sqrt{\tau} x_{i}
$$

So $d z_{i}=\sigma_{i} \sqrt{\tau} d x_{i}$.
Recalling the relationship between $q$ and $u$ described in (36), we therefore have

$$
\begin{aligned}
& q(t, y) \\
& =\frac{1}{\sqrt{2 \pi}} \sum_{i=1}^{n} \int_{-\infty}^{\infty} e^{-x_{i}^{2} / 2} \\
& \quad \times g\left(0, \ldots, 0, e^{\sigma_{i} \sqrt{T-t} x_{i}+y_{i}}, 0, \ldots, 0\right) d x_{i} .
\end{aligned}
$$

Hence, by formula (31), we have

$$
\begin{aligned}
& f(t, S) \\
& =\frac{e^{-r(T-t)}}{\sqrt{2 \pi}} \sum_{i=1}^{n} \int_{-\infty}^{\infty} e^{-x_{i}^{2} / 2} \\
& \times g\left(0, \ldots, 0, e^{\sigma_{i} \sqrt{T-t} x_{i}+\ln S_{i}-\left(r-\sigma_{i}^{2} / 2\right)(t-T)},\right. \\
& 0, \ldots, 0) d x_{i} .
\end{aligned}
$$

Since $e^{\ln S}=S$, then

$$
\begin{gathered}
f(t, S) \\
=\frac{e^{-r(T-t)}}{\sqrt{2 \pi}} \sum_{i=1}^{n} \int_{-\infty}^{\infty} e^{-x_{i}^{2} / 2} \\
\times g\left(0, \ldots, 0, S_{i} e^{\sigma_{i} \sqrt{T-t} x_{i}-\left(r-\sigma_{i}^{2} / 2\right)(t-T)}\right. \\
0, \ldots, 0) d x_{i} .
\end{gathered}
$$

In this way we proved Theorem 6.

\section{A Financial Example}

As an application, we consider the European call option. In Theorem 6, we have given the solution of the general B-S equation (17) which depends on the final data (18); that is, $f(T, s)=g(s)$. More specific, we take the final data $g(s)$ for the European call option as

$$
g(S)=g\left(S_{1}^{-k_{1}}, S_{2}^{-k_{2}}, \ldots, S_{n}^{-k_{n}}\right)=\sum_{i=1}^{n}\left(S_{i}-K_{\mathrm{i}}\right)^{+},
$$

where $S_{i}>0$ and $K_{i}>0$ are the strike price of $S_{i}$. Then we have the following corollary from Theorem 6 .

Corollary 7. For the European call option, the solution to the general Black-Scholes value problem (17) with the final data (48) is given by

$$
\begin{aligned}
f(t, S)= & \sum_{i=1}^{n} S_{i} \Phi\left(-A_{i}+\sigma_{i} \sqrt{T-t}\right) \\
& -e^{-r(T-t)} \sum_{i=1}^{n} K_{i} \Phi\left(-A_{i}\right)
\end{aligned}
$$


where

$$
\begin{gathered}
-A_{i}=\frac{\left(r-\sigma_{i}^{2} / 2\right)(T-t)+\ln \left(S_{i} / K_{i}\right)}{\sigma_{i} \sqrt{T-t}}=: d_{2}, \\
-A_{i}+\sigma_{i} \sqrt{T-t}=\frac{\left(r+\sigma_{i}^{2} / 2\right)(T-t)+\ln \left(S_{i} / K_{i}\right)}{\sigma_{i} \sqrt{T-t}}=: d_{1},
\end{gathered}
$$

that is,

$$
f(t, S)=\sum_{i=1}^{n} S_{i} \Phi\left(d_{1}\right)-e^{-r(T-t)} \sum_{i=1}^{n} K_{i} \Phi\left(d_{2}\right)
$$

In particular,

$$
f(0, S)=\sum_{i=1}^{n} S_{i} \Phi\left(d_{1}\right)-e^{-r T} \sum_{i=1}^{n} K_{i} \Phi\left(d_{2}\right) .
$$

Proof. For a European call option, we infer that

$$
S_{i} e^{\sigma_{i} \sqrt{T-t} x_{i}-\left(r-\sigma_{i}^{2} / 2\right)(t-T)}>K_{i}
$$

Dividing (53) by $S_{i}$ and taking the $\ln$, we get

$$
\sigma_{i} \sqrt{T-t} x_{i}-\left(r-\frac{\sigma_{i}^{2}}{2}\right)(t-T)>\ln \frac{K_{i}}{S_{i}}
$$

that is,

$$
x_{i}>\frac{\ln \left(K_{i} / S_{i}\right)-\left(r-\sigma_{i}^{2} / 2\right)(T-t)}{\sigma_{i} \sqrt{T-t}}=: A_{i} .
$$

Hence, from (30) and (48), it follows that

$$
\begin{aligned}
f(t, S)= & \frac{e^{-r(T-t)}}{\sqrt{2 \pi}} \\
& \times \sum_{i=1}^{n} \int_{A_{i}}^{\infty} e^{-x_{i}^{2} / 2} S_{i} e^{\sigma_{i} \sqrt{T-t} x_{i}-\left(r-\sigma_{i}^{2} / 2\right)(t-T)} d x_{i} \\
& -\frac{e^{-r(T-t)}}{\sqrt{2 \pi}} \sum_{i=1}^{n} K_{i} \int_{A_{i}}^{\infty} e^{-x_{i}^{2} / 2} d x_{i} \\
= & \frac{e^{-r(T-t)}}{\sqrt{2 \pi}} \sum_{i=1}^{n} \int_{A_{i}}^{\infty} S_{i} e^{\left(r-\sigma_{i}^{2} / 2\right)(T-t)} e^{-x_{i}^{2} / 2+\sigma_{i} \sqrt{T-t} x_{i}} d x_{i} \\
& -\frac{e^{-r(T-t)}}{\sqrt{2 \pi}} \sum_{i=1}^{n} K_{i} \int_{A_{i}}^{\infty} e^{-x_{i}^{2} / 2} d x_{i}
\end{aligned}
$$

$$
\begin{aligned}
= & \frac{1}{\sqrt{2 \pi}} \sum_{i=1}^{n} \int_{A_{i}}^{\infty} S_{i} e^{-\left(\sigma_{i}^{2} / 2\right)(T-t)} \\
& \times e^{-(1 / 2)\left(x_{i}-\sigma_{i} \sqrt{T-t}\right)^{2}+(1 / 2) \sigma_{i}^{2}(T-t)} d x_{i} \\
& -\frac{e^{-r(T-t)}}{\sqrt{2 \pi}} \sum_{i=1}^{n} K_{i} \int_{A_{i}}^{\infty} e^{-x_{i}^{2} / 2} d x_{i} \\
= & \frac{1}{\sqrt{2 \pi}} \sum_{i=1}^{n} \int_{A_{i}-\sigma_{i} \sqrt{T-t}}^{\infty} S_{i} e^{-z^{2} / 2} d z
\end{aligned}
$$

$$
\begin{gathered}
-\frac{e^{-r(T-t)}}{\sqrt{2 \pi}} \sum_{i=1}^{n} K_{i} \int_{A_{i}}^{\infty} e^{-x_{i}^{2} / 2} d x_{i} \\
=\sum_{i=1}^{n} S_{i} \frac{1}{\sqrt{2 \pi}} \int_{-\infty}^{-A_{i}+\sigma_{i} \sqrt{T-t}} e^{-x_{i}^{2} / 2} d x_{i}
\end{gathered}
$$$$
-e^{-r(T-t)} \sum_{i=1}^{n} K_{i} \frac{1}{\sqrt{2 \pi}} \int_{-\infty}^{-A_{i}} e^{-x_{i}^{2} / 2} d x_{i}
$$$$
=\sum_{i=1}^{n} S_{i} \Phi\left(-A_{i}+\sigma_{i} \sqrt{T-t}\right)
$$

$$
-e^{-r(T-t)} \sum_{i=1}^{n} K_{i} \Phi\left(-A_{i}\right),
$$

where $\Phi(t)$ is the probability distribution function of a standard Gaussion random variable $N(0,1)$; that is,

$$
\Phi(t)=\frac{1}{\sqrt{2 \pi}} \int_{-\infty}^{t} e^{-x^{2} / 2} d x, \quad t \in R .
$$

In this way, we have proved Corollary 7.

Remark 8. The above result is about the European call option. A similar representation to those from the above corollary in the European put option case can be obtained by taking $g(S)=\sum_{i=1}^{n}\left(K_{i}-S_{i}\right)^{+}, S_{i}>0$ for some fixed $K_{i}>0$.

\section{Conclusion}

In this paper, we have considered a financial market model with regime switching driven by geometric Lévy process. This financial market model is based on the multiple risky assets $S_{1}, S_{2}, \ldots, S_{n}$ driven by Lévy process. Itô formula and equivalent transformation methods have been used to solve this complicated financial market model. An example of the portfolio strategy and the final value problem to applying our method to the European call option has been given in the end of this paper.

\section{Conflict of Interests}

The authors declare that there is no conflict of interests regarding the publication of this paper. 


\section{Acknowledgment}

This work is supported by the National Natural Science Foundation of China (Grants no. 61075105 and no. 71371046).

\section{References}

[1] H. Markowitz, "Portfolio selection," Journal of Finance, vol. 7, pp. 77-91, 1952.

[2] D. Li and W.-L. Ng, "Optimal dynamic portfolio selection: multiperiod mean-variance formulation," Mathematical Finance, vol. 10, no. 3, pp. 387-406, 2000.

[3] B. Øksendal, Stochastic Differential Equations, Springer, 6th edition, 2005.

[4] X. Guo and Q. Zhang, "Optimal selling rules in a regime switching model," IEEE Transactions on Automatic Control, vol. 50, no. 9, pp. 1450-1455, 2005.

[5] M. Pemy, Q. Zhang, and G. G. Yin, "Liquidation of a large block of stock with regime switching," Mathematical Finance, vol. 18, no. 4, pp. 629-648, 2008.

[6] H. Wu and Z. Li, "Multi-period mean-variance portfolio selection with Markov regime switching and uncertain timehorizon," Journal of Systems Science \& Complexity, vol. 24, no. 1, pp. 140-155, 2011.

[7] H. Wu and Z. Li, "Multi-period mean-variance portfolio selection with regime switching and a stochastic cash flow," Insurance: Mathematics \& Economics, vol. 50, no. 3, pp. 371-384, 2012.

[8] J. Kallsen, "Optimal portfolios for exponential Lévy processes," Mathematical Methods of Operations Research, vol. 51, no. 3, pp. 357-374, 2000.

[9] D. Applebaum, Lévy Processes and Stochastic Calculus, vol. 93, Cambridge University Press, Cambridge, UK, 2004.

[10] N. Vandaele and M. Vanmaele, "A locally risk-minimizing hedging strategy for unit-linked life insurance contracts in a Lévy process financial market," Insurance: Mathematics \& Economics, vol. 42, no. 3, pp. 1128-1137, 2008.

[11] C. Weng, "Constant proportion portfolio insurance under a regime switching exponential Lévy process," Insurance: Mathematics \& Economics, vol. 52, no. 3, pp. 508-521, 2013.

[12] N. Bäuerle and A. Blatter, "Optimal control and dependence modeling of insurance portfolios with Lévy dynamics," Insurance: Mathematics \& Economics, vol. 48, no. 3, pp. 398-405, 2011.

[13] K. C. Yuen and C. Yin, "On optimality of the barrier strategy for a general Lévy risk process," Mathematical and Computer Modelling, vol. 53, no. 9-10, pp. 1700-1707, 2011. 


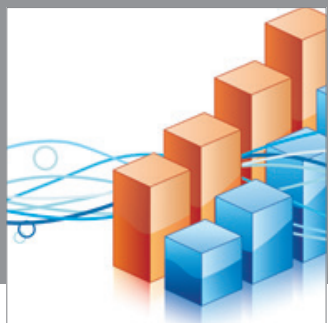

Advances in

Operations Research

mansans

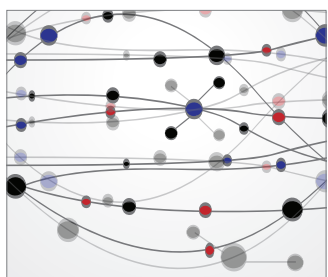

The Scientific World Journal
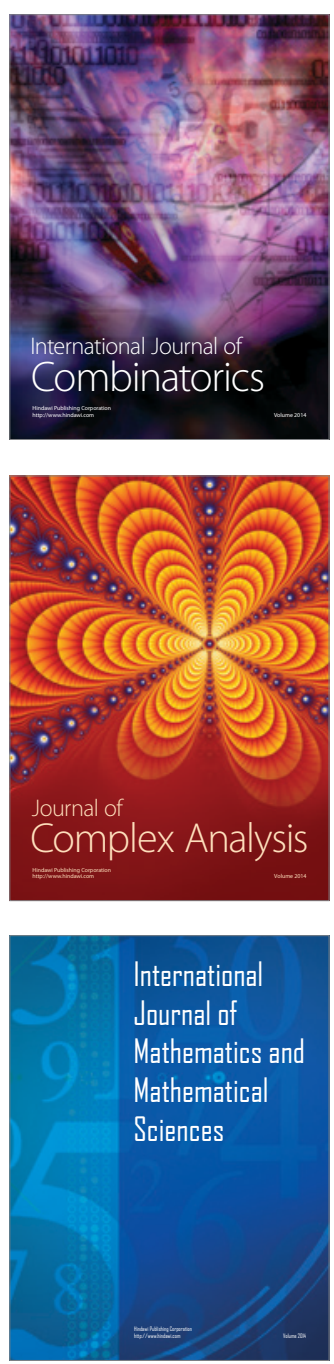
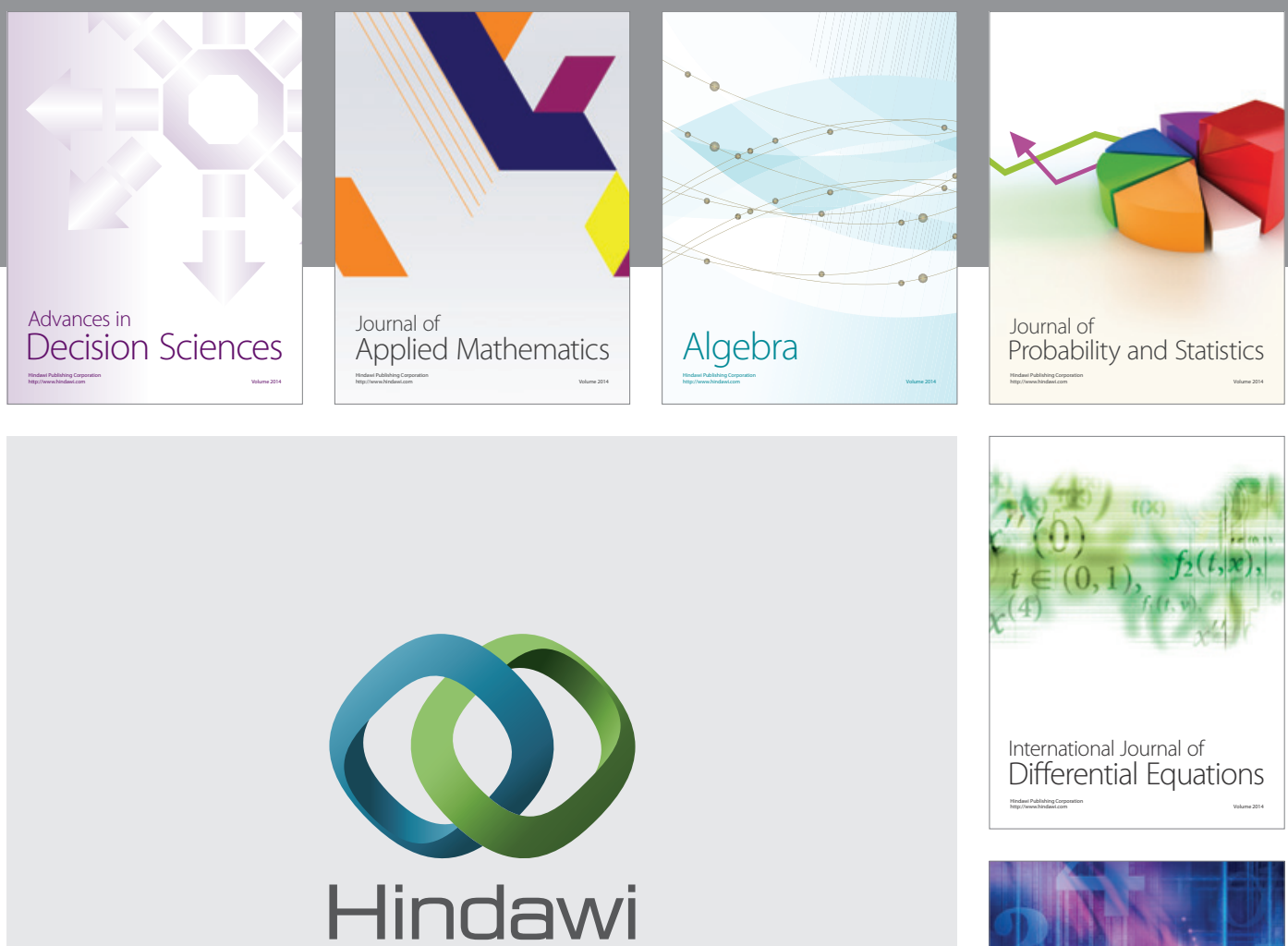

Submit your manuscripts at http://www.hindawi.com
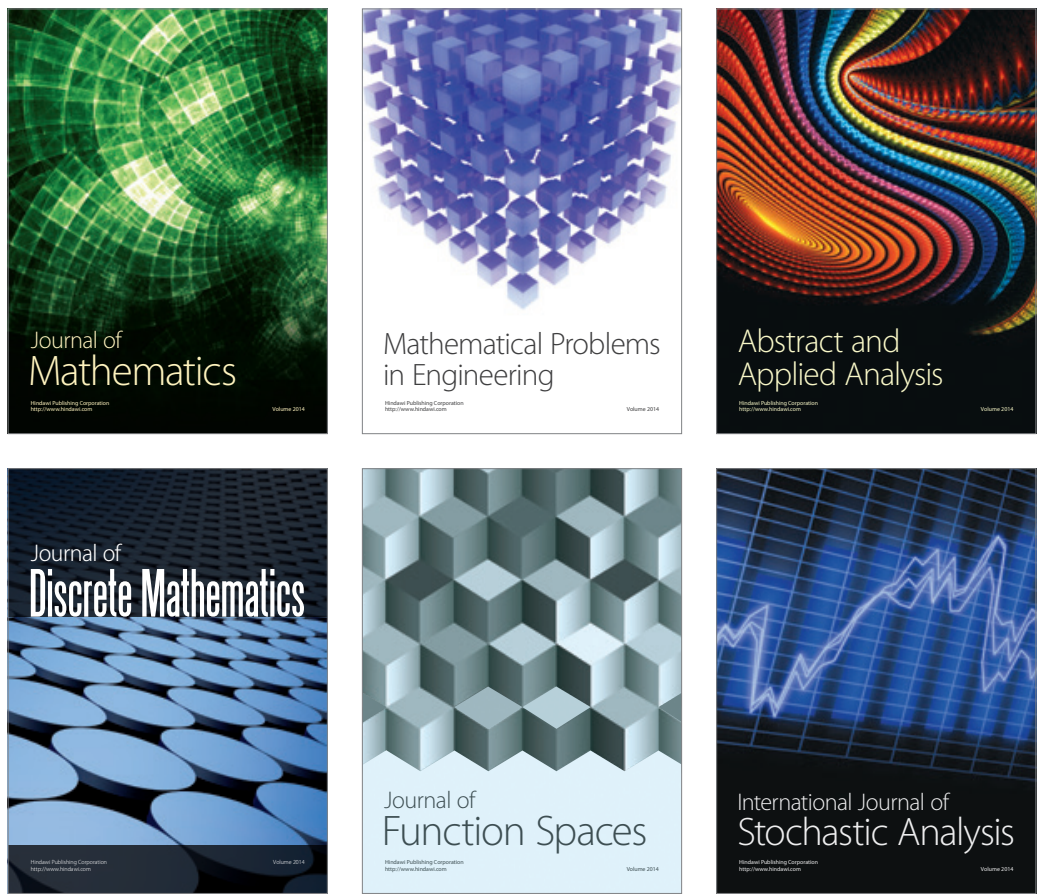

Journal of

Function Spaces

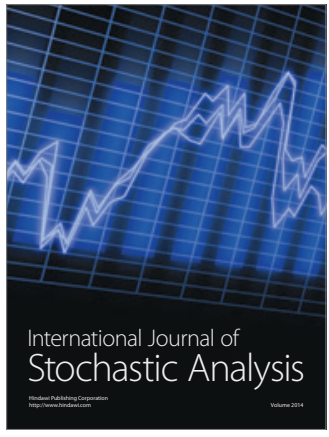

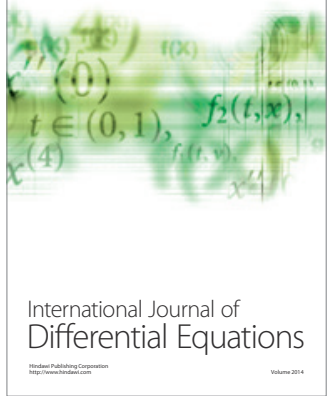
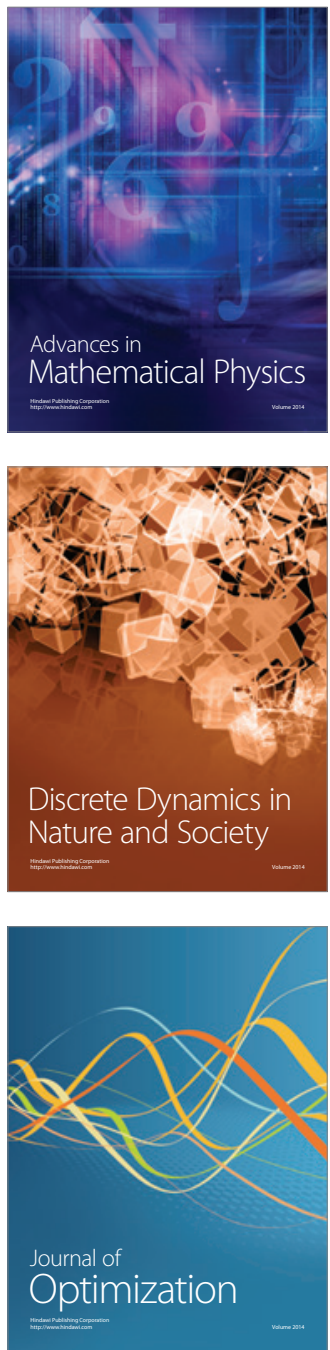